\title{
Highly accreting quasars: a tool for cosmology?
}

\author{
P. Marziani ${ }^{1}$, C. A. Negrete ${ }^{2}$, D. Dultzin ${ }^{2}$, \\ M. L. Martinez-Aldama ${ }^{3}$ A. Del Olmo ${ }^{3}$, D. Esparza ${ }^{2}$, \\ J. W. Sulentic ${ }^{3}$, M. D'Onofrio ${ }^{4}$, G. M. Stirpe ${ }^{5}$, E. Bon ${ }^{6}$ and N. Bon ${ }^{6}$ \\ ${ }^{1}$ INAF, Osservatorio Astronomico di Padova, Italy \\ email: paola.marziani@oapd.inaf.it \\ ${ }^{2}$ Instituto de Astronomía, UNAM, Mexico \\ ${ }^{3}$ IAA (CSIC), Granada, Spain \\ ${ }^{4}$ Università di Padova, Italy \\ ${ }^{5}$ INAF, Osservatorio Astronomico di Bologna, Italy \\ ${ }^{6}$ Belgrade Observatory, Serbia
}

\begin{abstract}
Highly accreting quasars are possible cosmological probes, as their Eddington ratio is expected to saturate toward values of order unity. We present preliminary estimates of redshiftindependent source luminosities and the Hubble diagram for quasars in the redshift range 0.1 $\lesssim z \lesssim 2.6$.
\end{abstract}

Keywords. quasars: general, quasars: emission lines, line: profiles, cosmological parameters, distance scale, dark matter

\section{Introduction}

Several new techniques have been proposed in recent years to use quasars as redshiftindependent distance indicators with the goal of deriving $\Omega_{\mathrm{M}}$ and $\Omega_{\Lambda}$. One of the most promising methods involves highly accreting quasars (xA for extreme accretors or extreme Population A) which may be considered as "Eddington standard candles:" their Eddington ratio is expected to converge toward values of order unity, with low scatter (Marziani \& Sulentic 2014). Using optical/UV spectral line widths as virial broadening estimators, we are then able to derive the black hole mass and a luminosity estimate avoiding the use of scaling laws. Highly accreting quasars are recognized from diagnostic ratios in their rest-frame UV and optical spectra. They yield a preliminary Hubble diagram with SDSS and dedicated Gran Telescopio Canarias (GTC) data.

\section{Highly accreting quasars: selection and cosmological exploitation}

The optical, UV, and X properties of quasars can be organized along a sequence defined by FeII prominence $R_{\mathrm{FeII}}=I(\mathrm{FeII} \lambda 4570) / I(\mathrm{H} \beta)$ and FWHM of broad $\mathrm{H} \beta$ (the optical plane of the "eigenvector 1 "). The optical criterion $R_{\mathrm{FeII}} \gtrsim 1$ is found equivalent to a UVbased criterion: (a) I(AliII $\lambda 1860) / \mathrm{I}(\mathrm{SiII}] \lambda 1892) \gtrsim 0.5$, (b) $I(\operatorname{SiII}] \lambda 1892) / \mathrm{I}(\mathrm{CIII}] \lambda 1909) \gtrsim 1$ which can be applied to high- $z$ quasars (Marziani \& Sulentic 2014). We are currently carrying out an analysis of survey data and of data collected from dedicated observations at GTC to obtain high-S/N spectra of xA sources.

It is possible to link accretion luminosity $L$ and line width $(\delta v \equiv \mathrm{FWHM}$ of $\mathrm{H} \beta$ or of the intermediate ionization lines AlıII $\lambda 1860$ and SiIII] 1892$)$ through multiplicative terms that can be assumed to have a small dispersion around an average value. Their product $\mathcal{L}_{0}$ can be assumed equal for all xA sources as a first approximation. The equation 


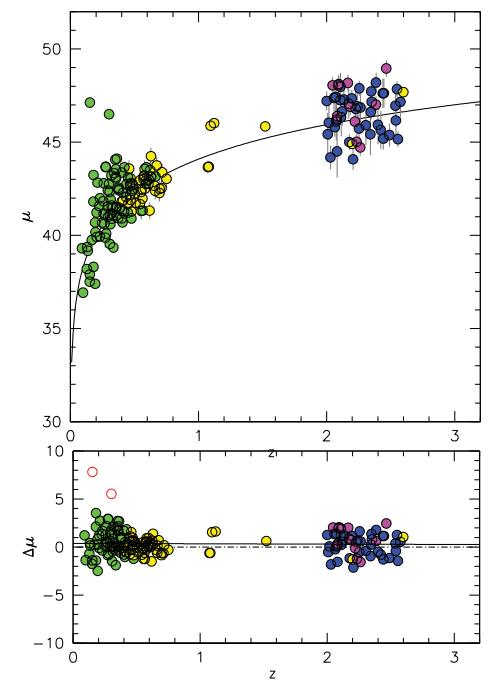

Figure 1. Preliminary Hubble diagram obtained from the analysis of the Marziani \& Sulentic (2014) data (yellow: $\mathrm{H} \beta$, navy blue: AlıII $\lambda 1860$ and SiIII] $\lambda 1892$ ) supplemented by new $\mathrm{H} \beta$ measurements from the SDSS (green) and from GTC observations (magenta). Red circles indicate outliers whose profile appears composite at a second inspection, suggesting the presence of a strong broadening component not associated with virial motions. The value of $\log \mathcal{L}_{0} \approx 45.06$ was used in the computation of the distance modulus $\mu$ for this work. The lower panel shows the distance modulus residuals with respect to concordance cosmology. The filled lines represents a lsq fit to the residuals as a function of $z$. Its slope $(b \approx-0.035 \pm 0.130)$ is not significantly different from 0 , indicating good statistical agreement between luminosities derived from concordance cosmological parameters and from the virial equation.

$L=\mathcal{L}_{0}(\delta v)^{4}$ was derived assuming virial line broadening, broad line region size scaling with luminosity as $\propto L^{\frac{1}{2}}$, as well as a well-defined $L / M$ ratio. If $L / M=$ const., then $L$ can be retrieved once the mass is known. The "virial luminosity" equation is the equivalent for a black hole system of the original Faber \& Jackson (1976) relation linking the luminosity and velocity dispersion of galaxies assuming a fixed mass-to-luminosity ratio.

The Hubble diagram of Fig. 1 includes 92 sources from Marziani \& Sulentic (2014), and is supplemented by 77 new SDSS low-luminosity xA sources at low $z$ covering the $\mathrm{H} \beta$ range, and by 10 new high- $z$ quasar observations with GTC of the fifty sources at redshift $2 \lesssim z<\lesssim 3$ that were observed with the OSIRIS camera at the GTC, covering the $\lambda 1900 \AA$ A blend. The sample $\mathrm{rms}$ is $\sigma_{\Delta \mu} \approx 1.1$ ( $\approx 0.44$ dex in luminosity), not improved with respect to the previous work, due to the slightly higher scatter found among the low-luminosity sources. The reanalysis of some quasars with $\Delta \mu \approx 3-4$ indicates that they are heavily affected by excess emission on the blue side of $\mathrm{H} \beta$ profile (represented by an unshifted Lorentz function). Outlying quasars show composite profile whose interpretation is currently not clear, and contamination by their host galaxy spectrum. However, the new data confirm the feasibility of the method, yielding a behavior consistent with concordance cosmology $\Omega_{\mathrm{M}} \approx 0.31$ and $\Omega_{\Lambda} \approx 0.69$ (Ade et al. 2016). Significant results require an increase in sample size, reduction of intrinsic scatter and skew as a function of $z$ (believed to be associated with orientation effects, and non-virial motions due to outflows), and precise knowledge of the flux ratio at 5100 and $1800 \AA$ if $\mathrm{H} \beta$ and the 1900 $\AA$ blend data are used together. Mock samples show that the method can in principle provide significant constraints especially on $\Omega_{\mathrm{M}}$, if applied to a quasar sample uniformly distributed over a redshift range $0.2 \lesssim z \lesssim 3$ (Marziani \& Sulentic 2014a).

\section{References}

Ade, P. A. R., Planck collaboration 2016, A\&A $A$, 594, 13

Faber, S. M. \& Jackson, R. E. 1976, ApJ, 204, 668

Marziani, P. \& Sulentic, J. W. 2014, MNRAS, 442, 1211

Marziani, P. \& Sulentic, J. W. 2014a, Adv. Spa. Res., 54, 1331

Wang, J.-M., Du, P., Valls-Gabaud, D., Hu, C., \& Netzer, H. 2013, Phys. Rev. Lett., 110, 081301 